\title{
Chebulinic acid derived from triphala is a promising antitumour agent in human colorectal carcinoma cell lines
}

\author{
Min Wang ${ }^{1}$, Yanru Li ${ }^{2}$ and Xianda $\mathrm{Hu}^{3^{*}}$ (D)
}

\begin{abstract}
Background: Triphala is an Ayurvedic rasayana formulation reputed for its antitumour activities, and chebulinic acid and chebulagic acid, along with other phenolic acids, have been proposed to be responsible for its effects.

Methods: In this study, the anti-proliferative activities of these agents were evaluated in colorectal carcinoma cell lines with three phenotypes exposed to several batches of triphala samples with different quantities of chebulinic acid and chebulagic acid. The pro-apoptotic and anti-migratory activities and the probable antitumour mechanisms of the more potent anti-proliferative phytochemical were also investigated.

Results: The results demonstrated that chebulinic acid, which exerts potent anti-proliferative, pro-apoptotic and anti-migratory effects, is a key molecule for maintaining the antitumour efficacy of triphala. The antitumour mechanism of chebulinic acid is probably related to the PI3K/AKT and MAPK/ERK pathways.

Conclusions: Chebulinic acid is not only a critical component of the anticancer activities of triphala but also a promising natural multi-target antitumour agent with therapeutic potential.
\end{abstract}

Keywords: Triphala, Colon cancer, Chebulinic acid, Chebulagic acid

\section{Background}

Colorectal cancer refers to malignant tumours that begin in cells of the large intestine, colon and rectum. Despite advances in diagnosis and treatment in past decades, colorectal cancer remains a significant health concern and substantial socioeconomic burden [1]. It has been reported that colorectal cancer is the third most common and fourth most deadly human malignancy globally [2].

Triphala is an Ayurvedic herbal formula consisting of three myrobalan fruits, namely, Phyllanthus emblica Linn., Terminalia chebula Retz., and Terminalia bellirica (Gaertn.) Roxb., in equal proportions [3]. Triphala was first recorded in the Ayurvedic text Charaka Samhita and has been used for various diseases for thousands of years [4]. Triphala has been used extensively not only in Ayurvedic medicine but also in many other traditional

\footnotetext{
* Correspondence: hellocean@hotmail.com

${ }^{3}$ Beijing Tibetan Hospital, China Tibetology Research Center, 218

Anwaixiaoguanbeili Street, Beijing 100029, Chaoyang, China

Full list of author information is available at the end of the article
}

medical practices that are influenced by Ayurveda, such as Tibetan, Thai and Unani medicines.

Recent clinical studies have proved that triphala has a wide spectrum of biological activities, including laxative, antimicrobial, immunomodulatory, and antioxidative activities, and is effective for constipation, gingivitis, arthritis, cataracts, and several other diseases or disorders [5-9]. Accumulating experimental evidence has also suggested that triphala is a promising herbal formulation for cancer therapy. It has been reported that triphala not only shows chemo-, radio- and oxidant-protective activities, which indicates that triphala has the potential to prevent oncogenesis [10-12], but also exhibits marked anti-proliferative and apoptosis-inducing properties against different tumour cell lines and animal models without causing damage to normal cells $[13,14]$. Moreover, triphala can regulate angiogenesis and epithelialto-mesenchymal transition to suppress tumour invasion and metastasis $[15,16]$.

Pharmaceutical analyses have revealed that triphala is rich in saponins, terpenoids, tannins, flavonoids and 
phenolic acids [17]. Among these compounds, ellagitannins and tannin-related compounds, especially gallic acid, ellagic acid, chebulinic acid and chebulagic acid, are considered the major constituents of the bioactivities of triphala [18-20]. However, the poor drug absorption and low bioavailability of gallic acid and ellagic acid has led to an increasing number of studies focused on chebulic ellagitannins [21, 22]. Both chebulinic acid and chebulagic acid have been demonstrated to contribute to the antitumour activities of triphala, but chebulinic acid shows higher antitumour activities than gallic acid, ethyl gallate, luteolin, and tannic acid against a human osteosarcoma cell line in vitro [23]. It has also been reported that chebulinic acid has the highest antioxidative activity among all the constituents of triphala [24]. Nevertheless, it remains difficult to determine whether chebulic ellagitannins are an important antitumour component, since interactions among the complex chemical components of triphala could result in synergistic or inhibitory effects that may influence antineoplastic activity. Previously, we obtained a batch of inferior triphala with a desired signature characterized by lower contents of chebulinic acid and chebulagic acid, which provides us with an opportunity to evaluate whether chebulinic acid is the major antitumour constituent of triphala.

\section{Methods}

\section{Drugs and chemicals}

The superior triphala preparation, which contains large amounts of chebulinic acid and chebulagic acid, was manufactured by Dabur India Ltd. (Alwar, India) with a batch number of AL1675, while the inferior triphala preparation containing lower levels of chebulinic acid and chebulagic acid was provided by Beijing Tibetan Hospital, China Tibetology Research Center. Standard analytical grade chebulinic acid and chebulagic acid were procured from Shanghai Yuanye Biological Technology (Shanghai, China).

\section{Preparation of triphala extract}

The superior and inferior triphala extracts were prepared following the same procedure. Finely powdered triphala was extracted by ultrapure water with refluxing for $1 \mathrm{~h}$. The extract solution was centrifuged at $4000 \mathrm{rpm}$ for $15 \mathrm{~min}$ at room temperature. Then, the supernatant was filtered through a $0.45 \mu \mathrm{m}$ membrane filter (Merck Millipore Ltd., Cork, Ireland) to remove particulate matter. The solvent was removed by rotary evaporation followed by freeze-drying. The achieved extract powder was weighed and stored at $-20^{\circ} \mathrm{C}$. The yields of the superior and inferior triphala extracts were 6.85 and $7.25 \%$, respectively.
High-performance liquid chromatography (HPLC) analysis The HPLC analysis was performed on a Waters Alliance 2695 system (Waters Corp, Milford, USA) equipped with a Waters 2489 UV detector. The dried triphala extract was dissolved in water, injected into a Waters XBridge Shield $\mathrm{RP}_{18}$ column $(250 \mathrm{~mm} \times 4.6 \mathrm{~mm} \times 5 \mu \mathrm{m})$ and eluted with a linear gradient from 15 to $80 \%$ aqueous acetonitrile solution. The absorbance was detected at $254 \mathrm{~nm}$.

\section{Cell culture}

The human colorectal carcinoma cell lines HR8348, LoVo, and LS174T were purchased from the China Infrastructure of Cell Line Resources and cultured in RPMI 1640 medium (Corning Inc., Corning, USA) supplemented with $10 \%$ foetal bovine serum (FBS) (Corning) and $1 \%$ penicillin-streptomycin (Beyotime Biotechnology Inc., Nantong, China) in a standard humidified incubator (NuAire Inc., Plymouth, USA) at $37^{\circ} \mathrm{C}$ under a $5 \% \mathrm{CO}_{2}$ atmosphere.

\section{Cell proliferation assay}

To evaluate cytotoxicity, cell viability was assessed by cell counting kit (CCK)-8 assays following the manufacturer's protocol (Dojindo Inc., Kumamoto, Japan). Briefly, the colorectal carcinoma cells were seeded in 96-well plates at a density of $1 \times 10^{4}$ cells per well for $24 \mathrm{~h}$ and cultured with increasing concentrations of drugs $(25-150$ $\mathrm{mg} / \mathrm{L})$ or chemicals $(20-70 \mu \mathrm{mol} / \mathrm{L})$ for an additional $48 \mathrm{~h}$. The tetrazolium salt-based CCK-8 solution was then added to each well, and the cells were incubated for another $3 \mathrm{~h}$. The optical density (OD) was measured at $450 \mathrm{~nm}$.

\section{Cell apoptosis assay}

To examine apoptosis, DNA fragmentation immunoassays were performed using the Cell Death Detection ELISA Plus kit (Roche Diagnostics, Basel, Switzerland) according to the manufacturer's specifications. Briefly, the cells were seeded and cultured with the same approach described in section 2.5. After incubation, the cells were collected by trypsinization, resuspended in phosphate-buffered saline (PBS), lysed with lysis buffer and centrifuged successively. The supernatants containing cytoplasmic histone-associated DNA fragments were used for the quantitative sandwich enzyme immunoassay. The OD was determined at $405 \mathrm{~nm}$.

\section{Transwell migration assay}

To detect metastatic ability, Boyden chamber assays were performed using the Transwell system (Corning) according to the manufacturer's instructions. Briefly, $5 \times 10^{4}$ colorectal cancer cells in serum-free RPMI 1640 medium were added to the upper layer of the cell-permeable 
membrane of a 24-well Transwell plate, while the lower chamber was filled with complete medium with FBS. Following $6 \mathrm{~h}$ of incubation, the cells that migrated through the micropores of the membrane to the lower chamber of the Transwell plate were stained using $0.1 \%$ crystal violet. The OD was determined at $630 \mathrm{~nm}$.

\section{Western blotting assay}

The colorectal carcinoma cells were cultured in $10 \mathrm{~cm}$ petri dishes and grown to approximately half confluence and then treated with chebulinic acid at the $\mathrm{IC}_{50}$ concentration for $48 \mathrm{~h}$. The cultured cells were collected and lysed in radioimmunoprecipitation assay (RIPA) buffer supplemented with protease inhibitor cocktail and phosphatase inhibitor cocktail (Sigma-Aldrich Corp., St Louis, USA). The concentration of extracted protein was measured using a Pierce BCA Protein Assay Kit (Thermo Fisher Scientific, Waltham, MA). After sodium dodecyl sulfate-polyacrylamide gel electrophoresis (SDS-PAGE) and transfer to a nitrocellulose membrane, the protein was reacted with monoclonal antibodies against AKT, ERK1/2 (Cell Signaling Technology, Danvers, USA) and caspase-3 (Abcam, Cambridge, UK) and subsequently labelled with secondary antibodies (Abcam), which were developed through enhanced chemiluminescence (ECL) using the Pierce ECL Plus Western Blotting Substrate (Thermo Fisher Scientific).

\section{Statistical analysis}

All data represent the mean \pm standard deviation (SD) of at least three independent experiments. Statistical analyses were performed by one-way ANOVA with the least significant difference (LSD) post hoc test for multiple comparisons using SPSS Statistics 19.0 software (IBM, Chicago, USA), and $p$ values less than 0.05 were considered statistically significant.

\section{Results}

The superior triphala preparation exhibits more potent anti-proliferative activities than the inferior triphala preparation against colorectal carcinoma cells

The cell viability effects of the superior and inferior triphala preparations on the human colorectal cancer cell lines HR8348, LoVo, and LS174T were investigated by a CCK-8 assay. The half maximal inhibition concentration $\left(\mathrm{IC}_{50}\right)$ values were calculated by probit analyses. The results showed that the superior triphala preparation had outstanding growth inhibition activities against all three tumour cells, which were significantly and positively correlated with the concentration (Fig. 2a). The $\mathrm{IC}_{50}$ values in HR8348, LoVo, and LS174T cells were $85.57 \pm 5.73$, $90.28 \pm 8.20$ and $84.50 \pm 3.56 \mathrm{mg} / \mathrm{L}$, respectively. In contrast, the inferior triphala preparation showed distinctly weaker cytotoxic effects on tumour cells (Fig. 2b), with
$\mathrm{IC}_{50}$ values of $127.24 \pm 6.09,129.28 \pm 8.12$ and $131.46 \pm$ $6.42 \mathrm{mg} / \mathrm{L}$, (Table 1) respectively, in the same cell lines.

The most important differentiating factor between the superior and inferior triphala preparations is the content of chebulinic acid and chebulagic acid

Quantitative analyses of the triphala compositions were performed by HPLC. The peaks were identified by aligning the retention time and peak shape with published data [25]. The amount of each component was estimated and compared using the area normalization method, and chebulinic acid and chebulagic acid were further quantified using the external standard method. The HPLC results illustrated that the compositions of the two triphala preparations were identical, whereas their relative contents were different (Fig. 1). The contents of chebulinic acid and chebulagic acid in the superior triphala preparation were 1.91 and 5.52 times higher than those in the inferior triphala preparation, respectively, while the levels of ellagic acid and gallic acid were 1.40 and 1.47 times lower than those in the inferior triphala preparation, respectively. There is sufficient evidence to prove that ellagic acid and gallic acid are antineoplastic. Predictably, the lower anti-proliferative activities of the inferior triphala preparation may result from the reduction in chebulinic acid and/or chebulagic acid content.

Chebulinic acid is more effective than chebulagic acid against the proliferation of colorectal carcinoma cells

The anti-proliferative activities of chebulinic acid and chebulagic acid were also investigated. The CCK-8 experiment results showed that although both chebulinic acid (Fig. 2e) and chebulagic acid (Fig. 2f) were able to restrain cell proliferation at low concentrations, the bioactivity of chebulinic acid is obviously higher than that of chebulagic acid. The $\mathrm{IC}_{50}$ values of chebulinic acid against HR8348, LoVo, and LS174T cells were $37.18 \pm 2.89,40.78 \pm 2.61$ and $38.68 \pm 2.12 \mu \mathrm{mol} / \mathrm{L}$, respectively, while the same values were $51.74 \pm 2.32$, $56.31 \pm 4.77$ and $53.53 \pm 0.65 \mu \mathrm{mol} / \mathrm{L}$, respectively, for chebulagic acid (Table 1 ).

Supplementation of the inferior triphala preparation with chebulinic acid, but not chebulagic acid, is able to significantly improve its anti-proliferative activities

To further determine the importance of chebulinic acid and/or chebulagic acid for the antitumour activities of triphala, chebulinic acid and chebulagic acid were added separately to the inferior triphala preparation in the correct proportion, according to the contents in the superior triphala preparation. The cytotoxic activities were detected by the aforementioned method. The results showed that the addition of chebulinic acid to the inferior triphala 


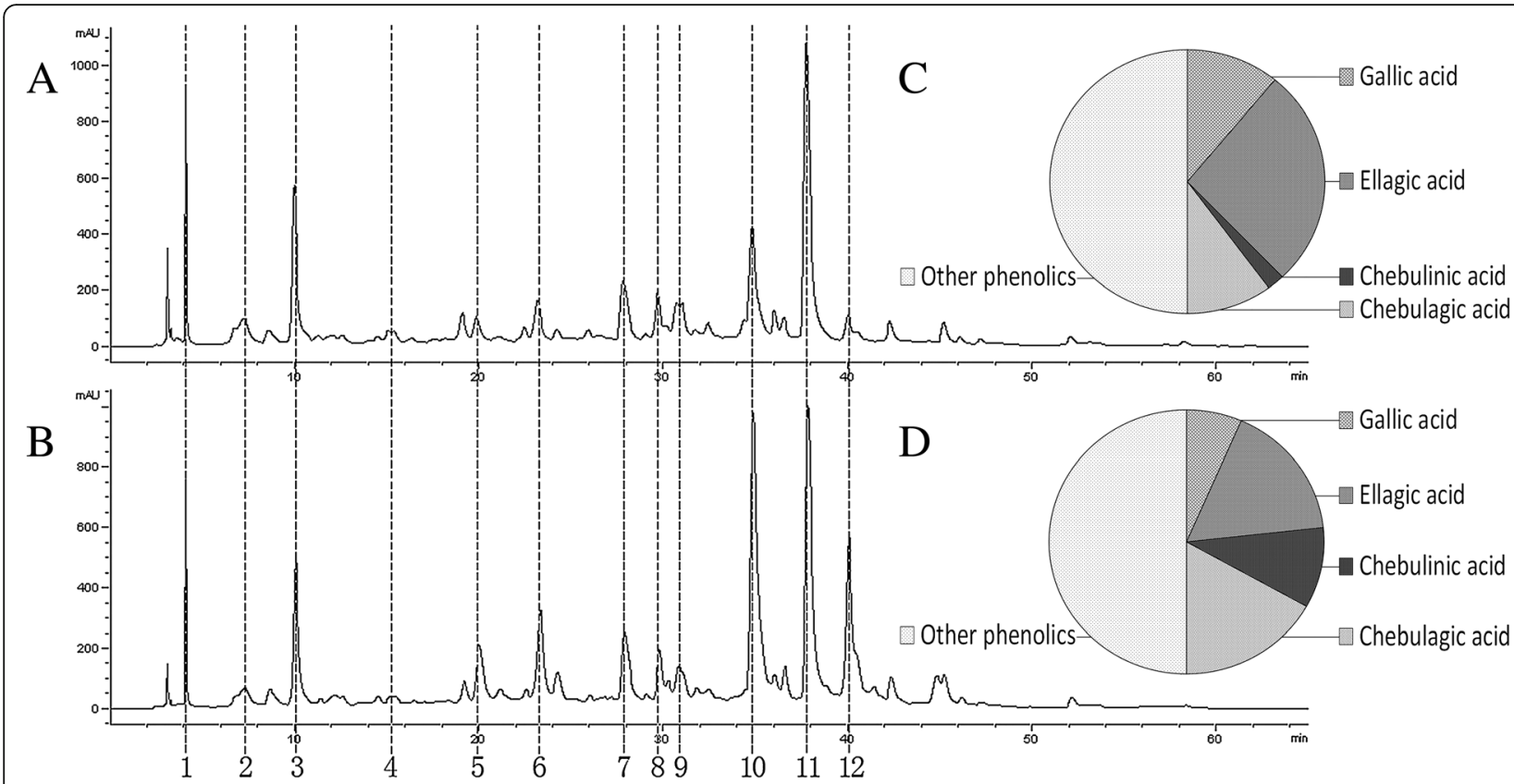

(1) Chebulic acid, (2) Glucogallin, (3) Gallic acid, (4) 1,6-di- $O$-galloyl- $\beta$-D-glucose, (5) $\alpha$-punicalagin, (6) $\beta$-punicalagin

(7) Corilagin (8) 3,4,6-tri-O-galloyl- $\beta$-D-glucose (9)Chebulanin (10) Chebulagic acid (11)Ellagic acid (12) Chebulinic acid

Fig. 1 Comparison of the contents of components between the superior and inferior triphala extracts, as determined by HPLC. a HPLC chromatogram of the superior triphala preparation; $\mathbf{b} \mathrm{HPLC}$ chromatogram of the inferior triphala preparation; $\mathbf{c}$ approximation of the contents of the superior triphala preparation based on the area percent by HPLC; and $\mathbf{d}$ approximation of the contents of the inferior triphala preparation based on the area percent by HPLC

preparation reinforces its anti-proliferative activities against the colorectal carcinoma cell lines HR8348, LoVo, and LS174T (Fig. 2c), with $\mathrm{IC}_{50}$ values of $81.19 \pm 6.05$, $87.34 \pm 4.62$ and $80.66 \pm 6.38 \mathrm{mg} / \mathrm{L}$, respectively. However, the addition of chebulagic acid alone was unable to significantly elevate the observed antitumour activities (Fig. 2d). The $\mathrm{IC}_{50}$ values of the inferior triphala preparation supplemented with chebulagic acid were $119.31 \pm 4.57,116.27 \pm 5.48$ and $118.82 \pm 4.20 \mathrm{mg} / \mathrm{L}$, respectively (Table 1 ). These results indicated that chebulinic acid is a vital component of triphala for restraining the proliferation of the above human colorectal carcinoma cell lines.

\section{Chebulinic acid is a promising pro-apoptotic and anti- metastatic agent}

The induction of apoptosis in the human colorectal cancer cell lines HR8348, LoVo, and LS174T after treatment with chebulinic acid was evaluated by enzyme-linked immunosorbent assay. The oligo-nucleosome enrichment of mono- and oligo-nucleosomes released into the cytoplasm was calculated as the ratio of the absorbance of treated cells to the absorbance of the corresponding negative control. The results demonstrated that chebulinic acid was able to induce programmed cell death significantly (Fig. 3a).
The inhibitory effects on motility were investigated by a Transwell migration assay. The effects of chebulinic acid on the migration of colorectal carcinoma cells were expressed as the ratio of the absorbance of treated cells to that of the control cells. The results revealed that chebulinic acid treatment induced a marked reduction in cellular migration towards the lower chamber (Fig. 3b).

\section{The antitumour effects of chebulinic acid are related to the activation of caspase- 3 and the downregulation of ERK pathways}

To investigate the molecular mechanism of chebulinic acid, Western blotting experiments were carried out to measure the levels of related proteins. The results showed that the degrees of p-ERK1/2 and p-AKT were decreased by 1.39 - and 1.32-fold in HR8348 cells, 1.96and 1.33-fold in LoVo cells, and 1.28- and 1.34-fold in LS174T cells, respectively, while the content of the cleaved caspase- 3 protein was increased by 3.26-, 1.40-, and 1.37-fold in HR8348, LoVo and LS174T cells, respectively (Fig. 4). This finding suggests that the antineoplastic mechanism of chebulinic acid may be related to the activation of caspase- 3 and the downregulation of the AKT and ERK pathways. 


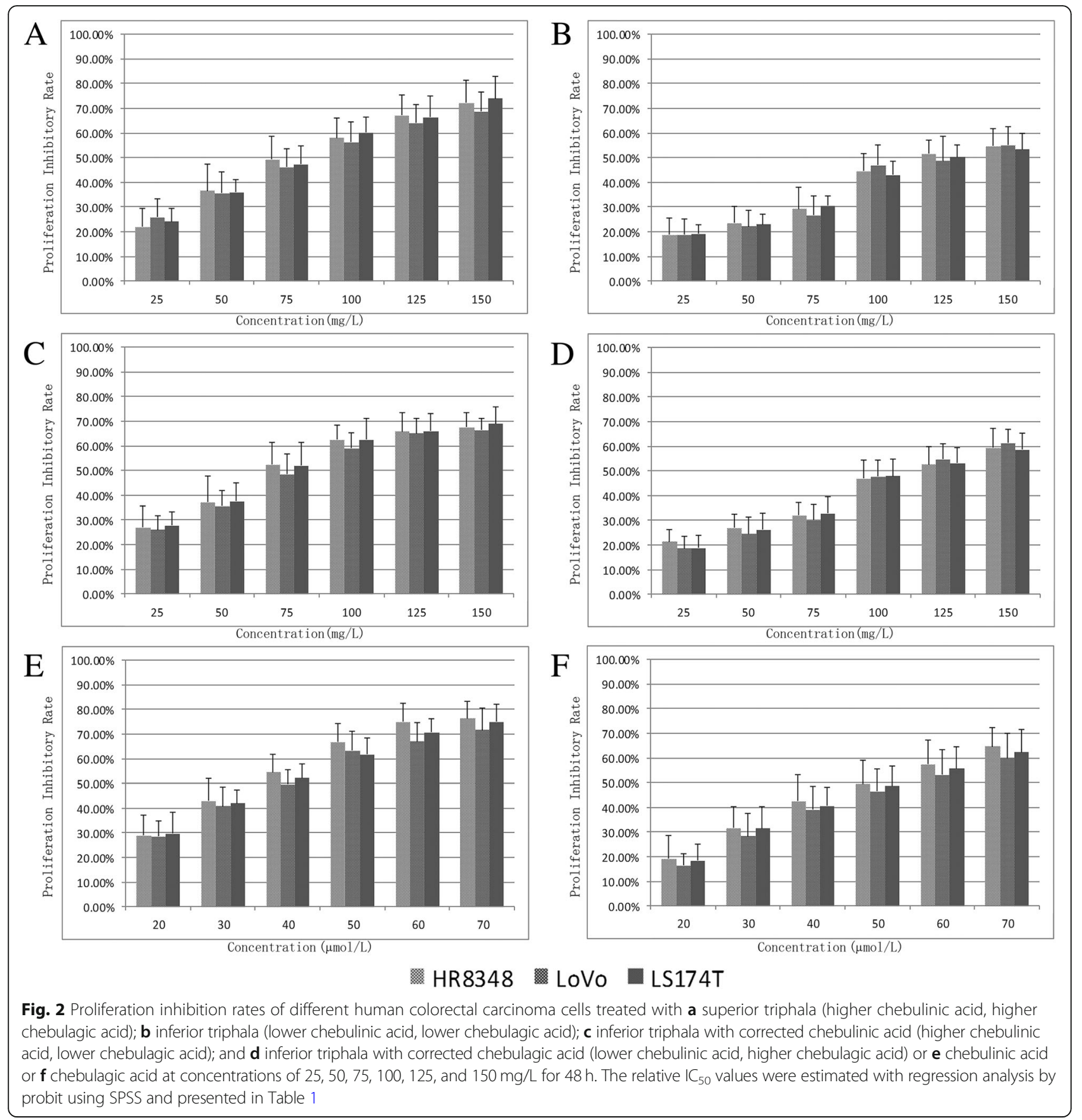

\section{Discussion}

Colorectal cancer is one of the most frequent and impactful cancers worldwide. Colorectal cancer also accounts for a significant and growing proportion of all cancer cases diagnosed in hospitals. Triphala, which is renowned for its colon cleansing and detoxification effects, has also been reported as a potent natural formulation that is effective against colorectal carcinoma stem cells and therefore has clinical value for the prevention and treatment of colon cancer [11]. In this study, the dose-dependent tumour-growth inhibitory activities of triphala on different human colorectal carcinoma cell lines were confirmed by CCK- 8 cytotoxic assays. However, a notable difference in antitumour effects was also observed between the two kinds of triphala, which was subsequently confirmed by HPLC analyses and attributed to variations in their component contents.

Among all discovered constituents of triphala, chebulinic acid, which exerts the most significant antitumour and antioxidant activities in vitro, has long been recognized as one of the most abundant and effective monomers [21, 22]. However, due to the synergistic and 

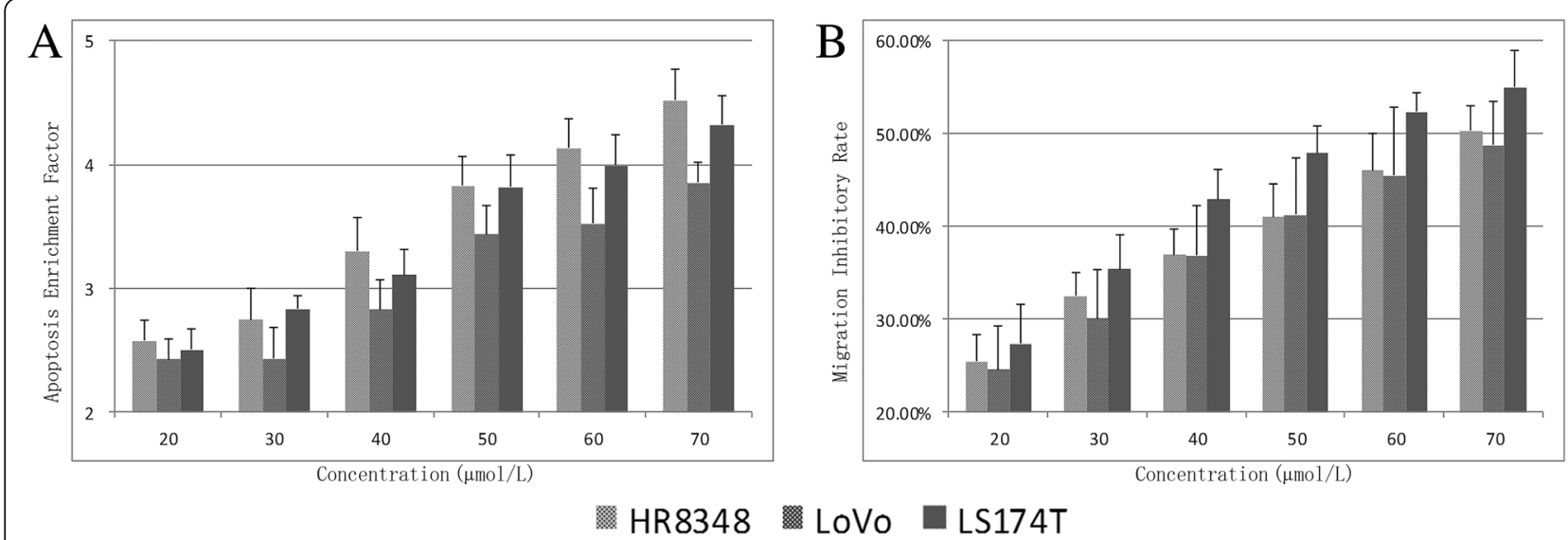

Fig. 3 Effects of chebulinic acid on cell apoptosis and migration in HR8348, LoVo, and LS174T cells. a Determination of apoptosis by a DNA fragmentation immunoassay. The enrichment factor was used as an index of apoptosis, which represents the level of DNA fragmentation in cells treated with chebulinic acid relative to that in untreated cells. $\mathbf{b}$ Examination of cell migration capacity through a Boyden chamber assay. The number of migrated cells was counted by absorbance after crystal violet staining. The inhibitory rates were calculated as percentages with respect to the control

antagonistic effects between the compounds present in the triphala, it is difficult to determine whether chebulinic acid is necessary for triphala bioactivity. Herein, we tested the anti-proliferative activities of a triphala preparation with higher chebulinic acid and chebulagic acid contents, a triphala preparation with lower chebulinic acid and chebulagic acid contents, and triphala preparations with corrected chebulinic acid or chebulagic acid contents in human colorectal carcinoma cells. The results demonstrated that the
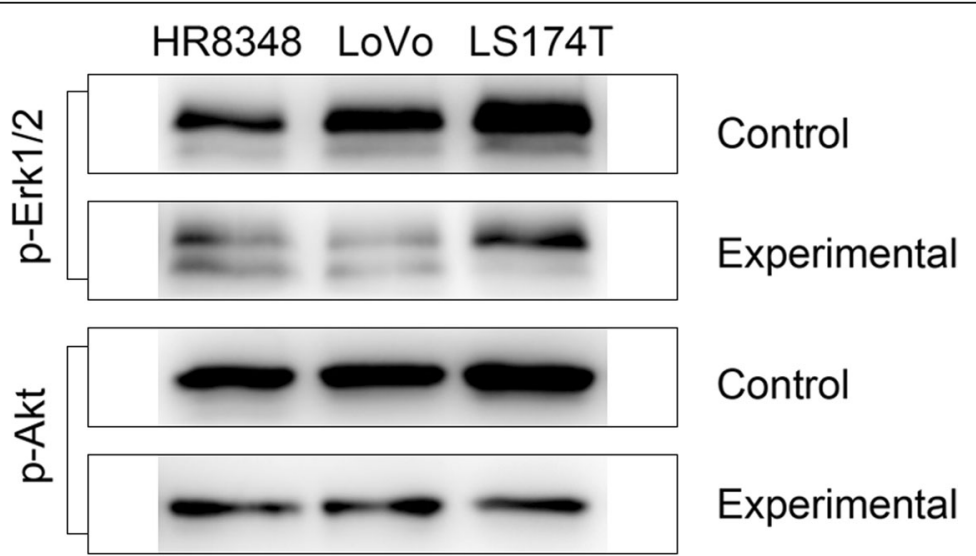

Control

Experimental

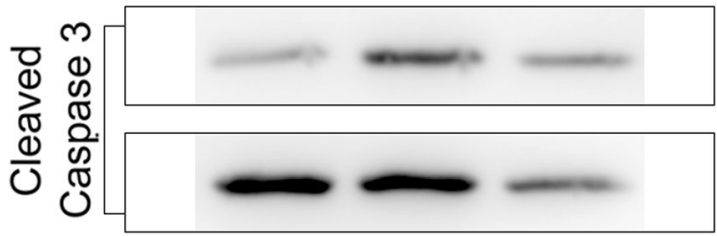

Control

Experimental

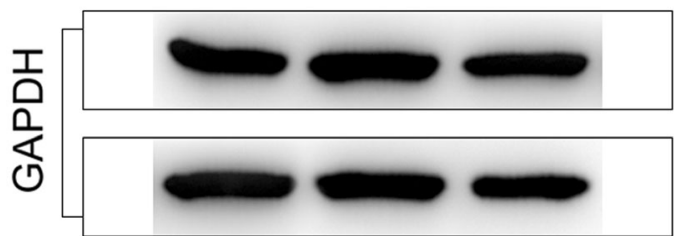

Control

\section{Experimental}

Fig. 4 Analyses of apoptosis-related proteins in HR8348, LoVo, and LS174T cells by Western blotting. The cells were treated with the IC $C_{50}$ of chebulinic acid for $48 \mathrm{~h}$. Phosphorylated ERK and AKT and cleaved caspase-3 were detected using the corresponding antibodies. GAPDH was used as a loading control 

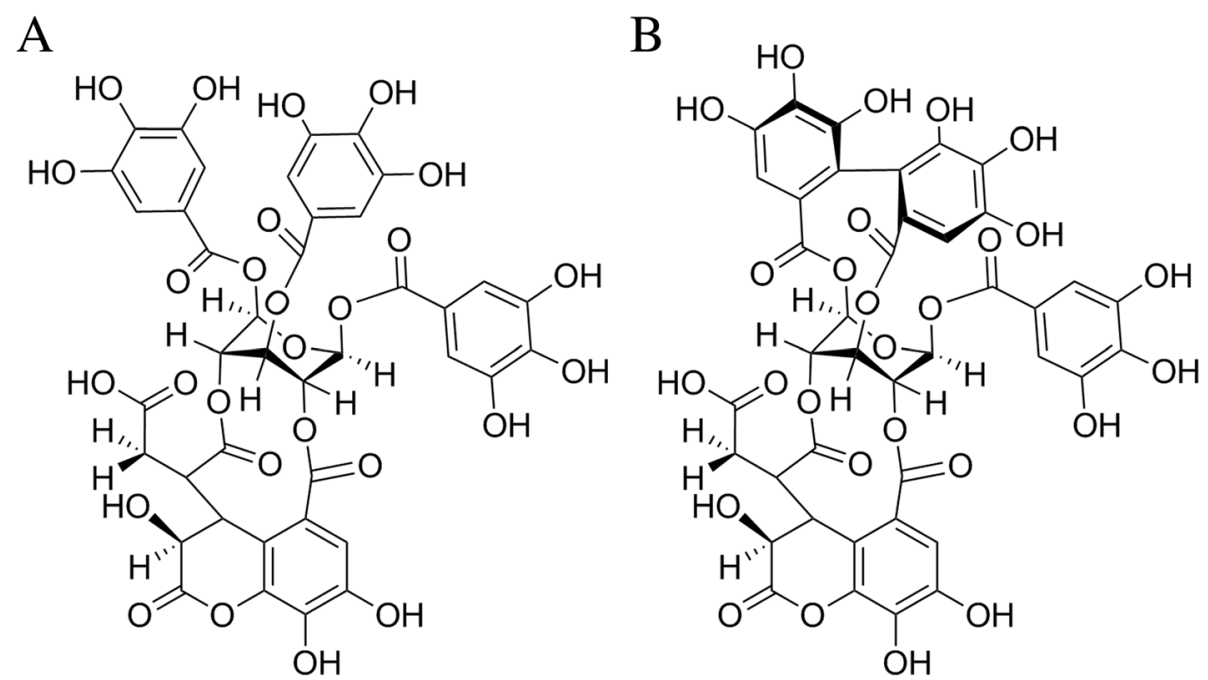

Fig. 5 Structures of chebulinic acid and chebulagic acid according to a previous study [26]. a chebulinic acid (1,3,6-tri-O-galloyl-2,4-O-chebuloyl$\beta$-D-glucopyranoside); b chebulagic acid (1-O-galloyl-2,4-O-chebuloyl-3,6-O-HHDP- $\beta$-D-glucose)

absence of chebulinic acid and chebulagic acid significantly affected the anti-growth effects of triphala, and these decreased anti-proliferative activities could be corrected by supplementation with chebulinic acid but not chebulagic acid. Moreover, chebulinic acid alone has been proven to be more effective than chebulagic acid. By comparing the above results, we concluded that chebulinic acid is a critical component that may play a dominant role in the anti-proliferation activity of triphala against colorectal cancer cells in vitro. Subsequent studies also revealed that chebulinic acid is a natural apoptosis-inductive and metastasis-inhibitive product.

Chebulinic acid and chebulagic acid share the same molecular backbone of a $\beta$-D-glucose residue linked to a chebuloyl moiety (Fig. 5). The difference between the molecules is that chebulinic acid has three free galloyl groups attached to the $\beta$-D-glucose residue, while chebulagic acid contains only one galloyl group and a hexahydrodiphenoyl (HHDP) group, which is assumed to be a substitution for the two galloyl groups [25]. The presence of the constrained HHDP group in chebulagic acid is considered to result in larger spatial hindrance and less molecular flexibility $[24,26]$. Chebulinic acid, in contrast, which has a more favourable structure for entering the binding cavities or catalytic pockets of target proteases or enzymes, usually shows more broadspectrum and potent biological activities (with the exception of chelation activity) [27, 28].

To date, chebulinic acid has been studied for its antioxidative, anti-pathogenic, anti-inflammatory, anti-fibrotic and antitumour activities [29-31]. Although the detailed mechanisms remain unclear, there is no doubt that chebulinic acid produces efficacy through multiple targets simultaneously. A series of tumour-associated proteins, such as vascular endothelial growth factor A (VEGF-A), small mothers against decapentaplegic homologue 3 (SMAD-3), matrix metalloprotease 2 (MMP-2), GATA-binding protein 2 (GATA2), and regulator of G protein signalling 7 and 8 (RGS-7/8), have been found or assumed to be related to the antitumour activities of chebulinic acid [15, 16, 32, 33]. The Western blotting results obtained in this study further proved that the cleavage of caspase- 3 was promoted while the phosphorylation of AKT and ERK was inhibited, which indicates that the PI3K/AKT and MAPK/ ERK pathways are also involved in the antitumour mechanism of chebulinic acid.

Table 1 Half maximal inhibition concentration (IC50) values of triphalas and chebulic ellagitannins

\begin{tabular}{lllllll}
\hline cell lines & A & B & C & D & E & F \\
\hline HR8348 & $85.57 \pm 5.73$ & $127.24 \pm 6.09$ & $81.19 \pm 6.05$ & $119.31 \pm 4.57$ & $37.18 \pm 2.89$ & $51.74 \pm 2.32$ \\
LoVo & $90.28 \pm 8.20$ & $129.28 \pm 8.12$ & $87.34 \pm 4.62$ & $116.27 \pm 5.48$ & $40.78 \pm 2.61$ & $56.31 \pm 4.77$ \\
LS174T & $84.50 \pm 3.56$ & $131.46 \pm 6.42$ & $80.66 \pm 6.38$ & $118.82 \pm 4.20$ & $38.68 \pm 2.12$ & $53.53 \pm 0.65$
\end{tabular}

The IC50 (mg/L) of superior triphala (A), inferior triphala (B), inferior triphala with chebulinic acid (C), inferior triphala with chebulagic acid (D), chebulinic acid (E) and chebulagic acid (F) 


\section{Conclusion}

Triphala is a phenolic-rich herbal formulation that is reputed for its anticancer activities. In this study, the obtained evidence indicates that chebulinic acid, as one of the major compounds in triphala, is required for the antitumour effects of triphala. The antineoplastic properties of chebulinic acid include anti-proliferation, pro-apoptosis, and anti-migration activities. The molecular mechanism is associated with the PI3K/AKT and MAPK/ ERK pathways. These results suggest the therapeutic potential of chebulinic acid for human colorectal cancer. However, the detailed molecular mechanism of the antitumour effects of chebulinic acid remains to be clarified.

\section{Abbreviations \\ CCK: Cell counting kit; GATA: GATA-binding protein; HHDP: Hexahydrodiphenoyl; $I_{50}$ : Half maximal inhibitory concentration; LSD: Least significant difference; MMP: Matrix metalloprotease; OD: Optical density; RGS: Regulator of G protein signalling; SD: Standard deviation; SMAD: Small mothers against decapentaplegic homologue; VEGF: Vascular endothelial growth factor}

\section{Acknowledgements}

The authors would like to thank the Ministry of Finance of the People's Republic of China, the China Postdoctoral Science Foundation, and Beijing Tibetan Hospital, China Tibetology Research Center for financially supporting this work.

\section{Funding}

This study was funded by the Ministry of Finance of the People's Republic of China, the China Postdoctoral Science Foundation (no. 2017 M610812), and Beijing Tibetan Hospital, China Tibetology Research Center under the Beijing Tibetan Hospital Research Grant Scheme (no. JC2016-01). The above funding agencies had no implication design of the study and collection, analysis, and interpretation of data and in writing the manuscript.

\section{Availability of data and materials}

The data sets supporting the results of this article are included within the article. The materials used in this research are available with the corresponding author.

\section{Authors' contributions}

This study was designed and performed by all three authors. The draft manuscript was prepared by MW and reviewed by $\mathrm{XH}$. All authors read and approved the final manuscript.

\section{Ethics approval and consent to participate}

Not applicable.

\section{Consent for publication}

Not applicable.

\section{Competing interests}

The authors declare that they have no competing interests.

\section{Publisher's Note}

Springer Nature remains neutral with regard to jurisdictional claims in published maps and institutional affiliations.

\section{Author details}

${ }^{1}$ Department of Rheumatology, Peking Union Medical College Hospital, Chinese Academy of Medical Sciences and Peking Union Medical College, Beijing, China. ${ }^{2}$ College of Chemistry and Molecular Engineering, Peking University, Beijing, China. ${ }^{3}$ Beijing Tibetan Hospital, China Tibetology Research Center, 218 Anwaixiaoguanbeili Street, Beijing 100029, Chaoyang, China
Received: 29 June 2017 Accepted: 17 December 2018

Published online: 27 December 2018

\section{References}

1. Jemal A, Siegel R, Ward E, Hao Y, Xu J, Murray T, Thun MJ. Cancer statistics, 2008. CA Cancer J Clin. 2008;58(2):71-96.

2. Ferlay J, Soerjomataram I, Dikshit R, Eser S, Mathers C, Rebelo M, Parkin DM, Forman D, Bray F. Cancer incidence and mortality worldwide: sources, methods and major patterns in GLOBOCAN 2012. Int J Cancer. 2015;136(5): E359-86.

3. Belapurkar P, Goyal P, Tiwari-Barua P. Immunomodulatory effects of triphala and its individual constituents: a review. Indian J Pharm Sci. 2014;76(6):467-75.

4. Mahdihassan S. Triphala and its Arabic and Chinese synonyms. Indian J Hist Sci. 1978;13(1):50-5.

5. Baliga MS, Meera S, Mathai B, Rai MP, Pawar V, Palatty PL. Scientific validation of the ethnomedicinal properties of the Ayurvedic drug Triphala: a review. Chin J Integr Med. 2012;18(12):946-54.

6. Mukherjee PK, Rai S, Bhattacharya S, et al. Clinical studies of 'triphala': a well known phytomedicine from India. Iran J Pharmacol Therapeut. 2006:5(1):51-4.

7. Baratakke SU, Raju R, Kadanakuppe S, Savanur NR, Gubbihal R, Kousalaya PS. Efficacy of triphala extract and chlorhexidine mouth rinse against plaque accumulation and gingival inflammation among female undergraduates: a randomized controlled trial. Indian J Dent Res. 2017;28(1):49-54.

8. Kalaiselvan S, Rasool M. Triphala exhibits anti-arthritic effect by ameliorating bone and cartilage degradation in adjuvant-induced arthritic rats. Immunol Investig. 2015:44(4):411-26.

9. Gupta SK, Kalaiselvan V, Srivastava S, Agrawal SS, Saxena R. Evaluation of anticataract potential of Triphala in selenite-induced cataract: in vitro and in vivo studies. J Ayurveda Integr Med. 2010;1(4):280-6.

10. Baliga MS. Triphala, Ayurvedic formulation for treating and preventing cancer: a review. J Altern Complement Med. 2010;16(12):1301-8.

11. Sharma A, Sharma KK. Chemoprotective role of triphala against 1,2dimethylhydrazine dihydrochloride induced carcinogenic damage to mouse liver. Indian J Clin Biochem. 2011;26(3):290-5.

12. Takauji Y, Miki K, Mita J, Hossain MN, Yamauchi M, Kioi M, Ayusawa D, Fujii M Triphala, a formulation of traditional Ayurvedic medicine, shows protective effect against X-radiation in HeLa cells. J Biosci. 2016:41(4):569-75.

13. Vadde R, Radhakrishnan S, Reddivari L, Vanamala JK. Triphala extract suppresses proliferation and induces apoptosis in human Colon Cancer stem cells via suppressing c-Myc/cyclin D1 and elevation of Bax/Bcl-2 ratio. Biomed Res Int. 2015;2015:649263.

14. Sandhya T, Lathika KM, Pandey BN, Mishra KP. Potential of traditional ayurvedic formulation, Triphala, as a novel anticancer drug. Cancer Lett. 2006;231(2):206-14.

15. Lu K, Chakroborty D, Sarkar C, Lu T, Xie Z, Liu Z, Basu S. Triphala and its active constituent chebulinic acid are natural inhibitors of vascular endothelial growth factor-a mediated angiogenesis. PLoS One. 2012;7(8):e43934.

16. Sivasankar S, Lavanya R, Brindha P, Angayarkanni N. Aqueous and alcoholic extracts of Triphala and their active compounds chebulagic acid and chebulinic acid prevented epithelial to mesenchymal transition in retinal pigment epithelial cells, by inhibiting SMAD-3 phosphorylation. PLoS One. 2015;10(3):e0120512

17. Avula B, Wang $\mathrm{YH}$, Wang $M$, Shen $\mathrm{YH}$, Khan IA. Simultaneous determination and characterization of tannins and triterpene saponins from the fruits of various species of Terminalia and Phyllantus emblica using a UHPLC-UV-MS method: application to triphala. Planta Med. 2013;79(2):181-8.

18. Russell LH, Mazzio E, Badisa RB, Zhu ZP, Agharahimi M, Millington DJ, Goodman CB. Differential cytotoxicity of triphala and its phenolic constituent gallic acid on human prostate cancer LNCap and normal cells. Anticancer Res. 2011;31(11):3739-45.

19. Pawar V, Lahorkar P, Anantha Narayana DB. Development of a RP-HPLC method for analysis of Triphala Curna and its applicability to test variations in Triphala Curna preparations. Indian J Pharm Sci. 2009;71(4):382-6.

20. Lu K, Basu S. The natural compound chebulagic acid inhibits vascular endothelial growth factor a mediated regulation of endothelial cell functions. Sci Rep. 2015:5:9642

21. Ferruzzi MG, Lobo JK, Janle EM, Cooper B, Simon JE, Wu QL, Welch C, Ho L, Weaver C, Pasinetti GM. Bioavailability of gallic acid and catechins from grape seed polyphenol extract is improved by repeated dosing in rats: implications for treatment in Alzheimer's disease. J Alzheimers Dis. 2009; 18(1):113-24. 
22. Seeram NP, Lee R, Heber D. Bioavailability of ellagic acid in human plasma after consumption of ellagitannins from pomegranate (Punica granatum L.) juice. Clin Chim Acta. 2004;348(1-2):63-8.

23. Saleem A, Husheem M, Härkönen P, Pihlaja K. Inhibition of cancer cell growth by crude extract and the phenolics of Terminalia chebula retz. Fruit. J Ethnopharmacol. 2002;81(3):327-36.

24. Klika KD, Saleem A, Sinkkonen J, Kähkönen M, Loponen J, Tähtinen P, Pihlaja $K$. The structural and conformational analyses and antioxidant activities of chebulinic acid and its thrice-hydrolyzed derivative, 2,4-chebuloyl- $\beta$-dglucopyranoside, isolated from the fruit of Terminalia chebula. ARKIVOC. 2004; (7):83-105.

25. Olennikov DN, Kashchenko NI, Chirikova NK. In vitro bioaccessibility, human gut microbiota metabolites and Hepatoprotective potential of Chebulic Ellagitannins: a case of Padma Hepaten formulation. Nutrients. 2015;7(10): 8456-77.

26. Pfundstein B, El Desouky SK, Hull WE, Haubner R, Erben G, Owen RW. Polyphenolic compounds in the fruits of Egyptian medicinal plants (Terminalia bellerica, Terminalia chebula and Terminalia horrida): characterization, quantitation and determination of antioxidant capacities. Phytochemistry. 2010;71(10):1132-48.

27. Patel K, Tyagi C, Goyal S, Jamal S, Wahi D, Jain R, Bharadvaja N, Grover A. Identification of chebulinic acid as potent natural inhibitor of $\mathrm{M}$. tuberculosis DNA gyrase and molecular insights into its binding mode of action. Comput Biol Chem. 2015;59:37-47.

28. Moilanen J, Karonen M, Tähtinen P, Jacquet R, Quideau S, Salminen JP. Biological activity of ellagitannins: effects as anti-oxidants, pro-oxidants and metal chelators. Phytochemistry. 2016;125:65-72.

29. Manosroi A, Jantrawut P, Akazawa H, Akihisa T, Manosroi J. Biological activities of phenolic compounds isolated from galls of Terminalia chebula Retz. (Combretaceae). Nat Prod Res. 2010;24(20):1915-26.

30. Chuang HY, Ng LT, Lin LT, Chang JS, Chen JY, Lin TC, Lin CC. Hydrolysable tannins of tropical almond show antifibrotic effects in TGF- $\beta 1$-induced hepatic stellate cells. J Sci Food Agric. 2011;91(15):2777-84.

31. Kesharwani A, Polachira SK, Nair R, Agarwal A, Mishra NN, Gupta SK. AntiHSV-2 activity of Terminalia chebula Retz extract and its constituents. chebulagic and chebulinic acids BMC Complement Altern Med. 2017;17(1):110.

32. Yi ZC, Wang Z, Li HX, Liu MJ, Wu RC, Wang XH. Effects of chebulinic acid on differentiation of human leukemia K562 cells. Acta Pharmacol Sin. 2004; 25(2):231-8.

33. Chandran U, Mehendale N, Tillu G, Patwardhan B. Network pharmacology of Ayurveda formulation Triphala with special reference to anti-Cancer property. Comb Chem High Throughput Screen. 2015;18(9):846-54.

Ready to submit your research? Choose BMC and benefit from:

- fast, convenient online submission

- thorough peer review by experienced researchers in your field

- rapid publication on acceptance

- support for research data, including large and complex data types

- gold Open Access which fosters wider collaboration and increased citations

- maximum visibility for your research: over $100 \mathrm{M}$ website views per year

At BMC, research is always in progress.

Learn more biomedcentral.com/submissions 